
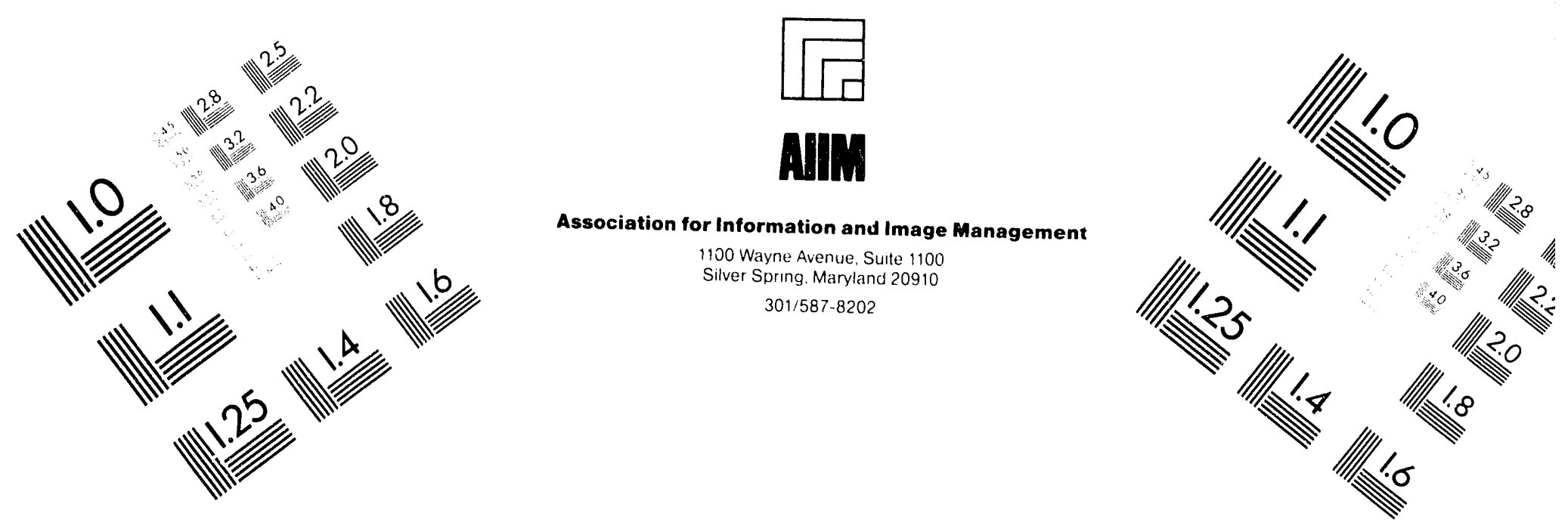

Centimeter

$\mid \begin{aligned} & 1 \\ & \mid\end{aligned}$ Inches
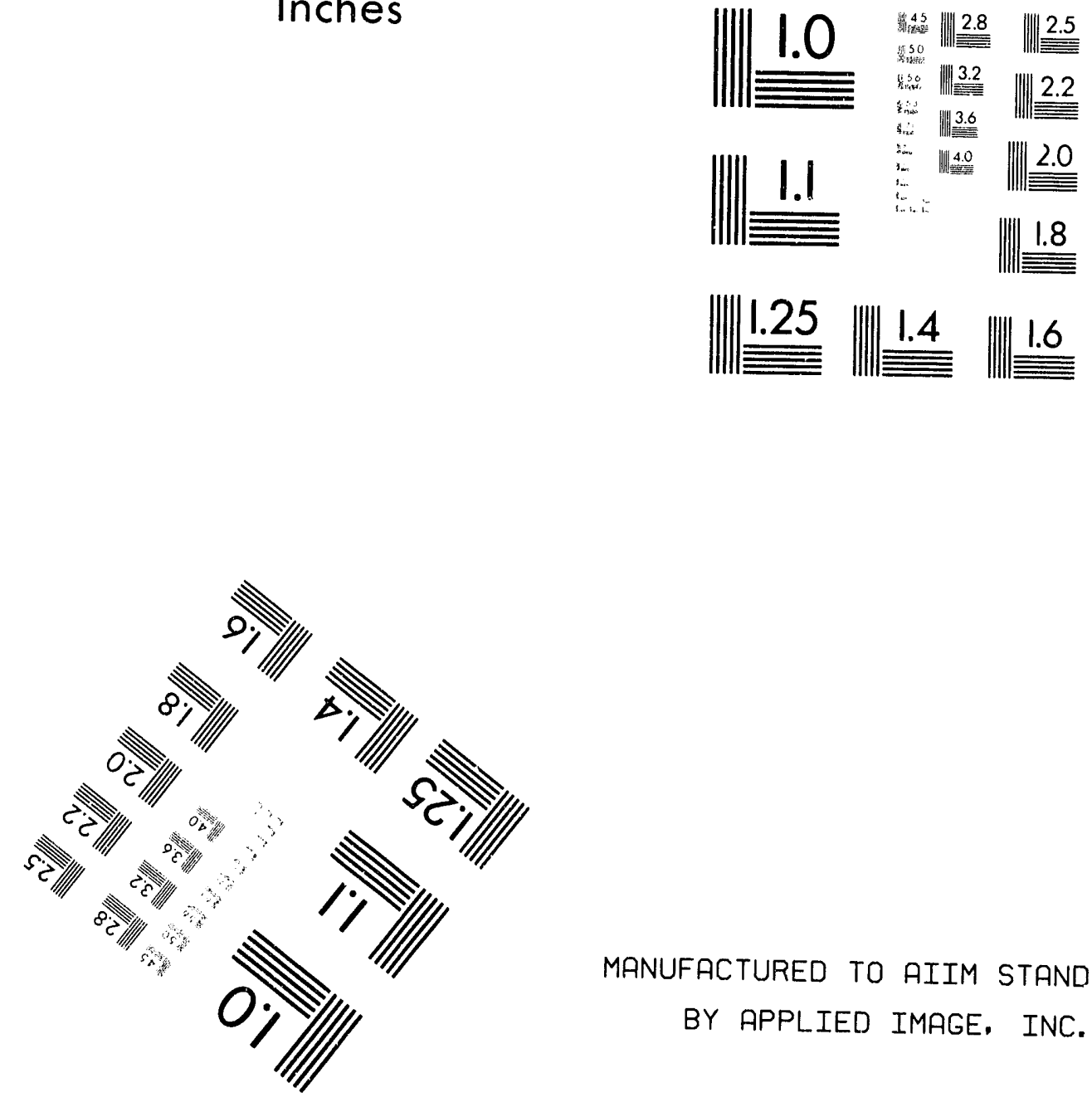

MANUFACTURED TO AIIM STANDARDS

BY APPLIED IMAGE, INC.

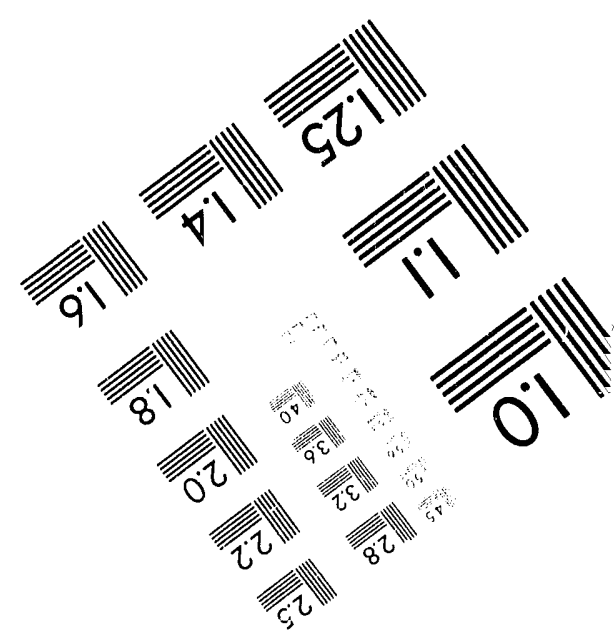



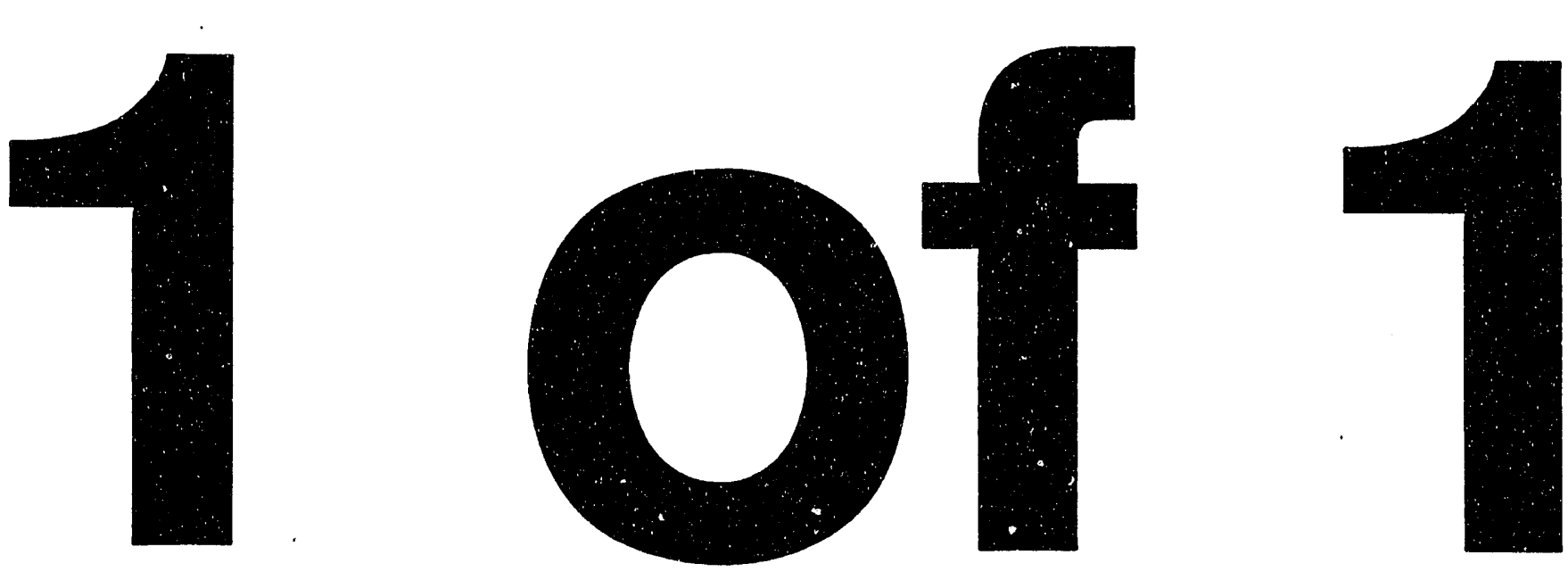
DOE/ER/40757--2

CPP-55

April 1993

\section{Avoiding Degenerate Coframes in an Affine Gauge Approach to Quantum Gravity}

by

Eckehard W. Mielke, J. Dermott McCrea, Yuval Ne'eman and Friedrich W. Hehl

Center for Particle Physics

University of Texas at Austin

Austin, Texas $\mathbf{7 8 7 1 2}$ 


\title{
Phys. Rev. D \\ AVOIDING DEGENERATE COFRAMES \\ IN AN AFFINE GAUGE APPROACH TO QUANTUM GRAVITY
}

by

\author{
Eckehard W. Mielke*\$, J. Dermott McCrea**, Yuval Ne'eman***\$+), \\ and Friedrich W. Hehl*\$
}

*) Institute for Theoretical Physics, University of Cologne, D(W)-5000 Köln 41, Germany

**) Department of Mathematical Physics, University College, Dublin 4, and Dublin Insítute for Advanced Studies, Dublin 4, Ireland

***) Raymond and Beverley Sackler Faculty of Exact Sciences, Tel-Aviv University, Tel-Aviv 69978, Israel

\begin{abstract}
In quant um models of gravity, it is surmized that configurations with degenerate coframes could occur during topology change of the underlying spacetime structure. However, the coframe is not the true Yang-Mills type gauge field of the translations, since it lacks the inhomogeneous gradient term in the gauge transformations. By explicitly restoring this "hidden" piece within the framework of the affine gauge approach to gravity, one can avoid the metric or coframe degeneracy which would otherwise interfere with the integrations within the path integral. This is an important advantage for quantization.

PACS numbers: $04.50 .+\mathrm{h}, 11.15 .-\mathrm{q}, 12.25 .+\mathrm{e}$.
\end{abstract}

-) On leave from the Center for Particle Physics, University of Texas, Austin, Texas 78712, USA

\$) Supported by the German-Israeli Foundation for Scientific Research \& Development (GIF), Jerusalem and Munich, project I-52-212.7/87.

+)Supported in part by DOE Grant DE-FG05-85-ER40200. 


\section{Introduction}

As it should be recognized by now, "...gravity is that field which corresponds to a gauge invariance with respect to displacement transformations", as Feynman [1] has put it. On a macroscopic scale, gravity is empirically rather well described by Einstein's general relativity theory (GR) which resides in a curved pseudo-Riemannian spacetime.

In a first order formalism, one introduces a local frame field (or vielbein), $e_{\alpha}=$ $e^{i}{ }_{\alpha} \partial_{i}$ which is expanded via the tetrad coefficients $e_{\alpha}^{i}$ in terms of the coordinate basis $\partial_{i}:=\partial / \partial x^{i}$, together with the coframe field or 1 -form basis $\vartheta^{\beta}=e_{j}{ }^{\beta} d x^{j}$, which is dual to the frame $\epsilon_{\alpha}$ with respect to the interior product: $\left.e_{\alpha}\right\rfloor \vartheta^{\beta}=e^{i}{ }_{\alpha} e_{i}{ }^{\beta}=\delta_{\alpha}^{\beta}$. In the Introduction the frame is chosen to be (pseudo-)orthonormal. Quite often, $\vartheta^{\alpha}$ is advocated as the translational gauge potential, although it does not transform inhomogeneously under local frame rotations, as is characteristic for a connection.

The Einstein-Cartan Lagrangian [2] is given by

$$
V_{\mathrm{EC}}=-\frac{1}{4 \ell^{2}} \stackrel{\circ}{R}^{\alpha 3} \wedge v^{\urcorner} \wedge \vartheta^{\delta} \eta_{\alpha \beta \gamma \delta}
$$

where $\stackrel{\circ}{R}^{\alpha \beta}=R^{[\alpha \beta]}$ is the curvature 2-form associated with the Lorentz connection 1-forms $\stackrel{\circ}{\Gamma}^{\alpha \beta}=\Gamma^{[\alpha \beta]}$ and $\eta_{\alpha \beta \gamma \delta}:=\sqrt{\left|\operatorname{det} o_{\alpha \beta}\right|} \epsilon_{\alpha \beta \gamma \delta}$ is the Levi-Civita tensor. Because of the orthonormality chosen, the local metric components read $o_{\alpha \beta}=$ $\operatorname{diag}(1,-1,-1,-1)$. In constructing macroscopic viable gravitational Lagrangians, the fundamental length $\ell$ needs to be identified with the Planck length $\ell_{\text {Planck }}$. Vacuum GR can be consistently recovered by imposing the constraint of vanishing torsion $T^{\alpha}:=D \vartheta^{a}$ via the addition of the Lagrange multiplier term $\mu_{\alpha} \wedge T^{\alpha}$ to (1.1). Then only the Belinfante-Rosenfeld symmetrized energy-momentum current occurs as a source of gravity, the contribution of the matter spin being subtracted out.

One avenue of quantizing gravity is to consider the functional integral

$$
\int \mathcal{D} \vartheta \mathcal{D} \stackrel{\circ}{\Gamma} \exp \left(i \int_{M} V_{\mathrm{EC}}\right)
$$

where a summation is understood over all inequivalent coframes $\vartheta:=\vartheta^{\alpha} P_{\alpha}$, Lorentz connections $\stackrel{\circ}{\Gamma}:=\stackrel{\circ}{\Gamma}^{\alpha \beta} \stackrel{\circ}{L}_{\alpha \beta}$, and spacetime topologies, as well. Since in GR the Lorentz connection $\stackrel{\circ}{\Gamma}^{\alpha \beta}$ is constrained by $T^{\alpha}=0$, Eq. (1.1) will become the Hilbert second order Lagrangian and an integration over all coframes is sufficient. In any case, this summation will also involve degenerate $\left(\operatorname{det} e_{j}{ }^{\beta}=0\right)$ or even vanishing coframes. This instance would induce the breakdown of any length measurement performed by means of the metric $g=o_{\alpha \beta} \vartheta^{\alpha} \otimes \vartheta^{\beta}$ and would signal the possible occurence of a topology change, as is argued in the interesting paper of Horowitz [3]. This also gives a flavor of some of the conceptual difficulties [4] not encountered in the quantization of internal Yang-Mills theories on a fixed spacetime background. 
Degenerate coframes are not only restricted to the realm of quantum gravity. In Ashtekar's reformulation of canonical GR, cf. [5], the "triad density", i.e. more precisely, the tangential 2-form $\underline{\vartheta}^{\alpha}$, is in fact allowed to become degenerate as a classical solution of Hamilton's equations. Moreover, in a first order formulation of topological 3D gravity [6], we uncovered a "dynamical symmetry" in which coframe and "Lorentz"-rotational connection (in three dimensions) become related ${ }^{(1)}$ to each other via $\vartheta^{A}=c \stackrel{\circ}{\Gamma}^{\star A}=\frac{c}{2} \eta^{A B C} \stackrel{\circ}{\Gamma}_{B C}$, where $A, B, C=0,1,2$ (or $=1,2,3$ for Euclidean signature). For $\stackrel{\circ}{\Gamma}_{A B} \rightarrow 0$, a degenerate coframe ("triad") will occur in this model. By regarding coframe and "Lorentz"-rotational connection as part of the Cartan connection

$$
\overline{\bar{\Gamma}}=\vartheta^{A} P_{A}+\stackrel{\circ}{\Gamma}^{B C} \stackrel{\circ}{L}_{B C},
$$

Witten [8] could show that the 3D Hilbert-Einstein Lagrangian can be absorbed in a Chern-Simons term for (1.3), thus facilitating the proof of the finiteness of the corresponding $3 \mathrm{D}$ quantum model.

Degenerate coframes, however, tend to jeopardize the coupling of gravity to matter fields. as exemplified by Dirac or Rarita-Schwinger fields. The basic reason is that the local frame $e_{\alpha}$, even if it still exists, is not invertible any more; i.e. the relation $\left.\epsilon_{\alpha}\right\rfloor \vartheta^{\beta}=\delta_{\alpha}^{\beta}$, which is needed in the formulation of the matter Lagrangian, would then be lost. In this paper, we want to resolve this riddle by demonstrating explicitly that $\vartheta^{a}$ is only part of the dimensionless translational gauge potential, if we use a Yang-Mills type gauge approach to the affine group, which includes the Poincaré group of elementary particle physics as subgroup. Thereby we also clarify the subtle relationship between infinitesimal translational gauge transformations and four one-parameter subgroups of diffeomorphism of spacetime, both regarded as acting actively.

\section{The rigid affine group $A(n, R)$}

In the flat $n$-dimensional affine space $R^{n}$, the rigid affine group $A(n, R):=R^{n} \otimes$ $G L(n, R)$ acts as the semidirect product of the group of $n$-dimensional translations and n-dimensional general linear transformations. Thus it is a generalization of the Poincaré group $P:=R^{4} \otimes S O(1.3)$, with the pseudo-orthogonal group $S O(1, n-1)$ being replaced by the general linear group $G L(n, R)$. In the following, we will work in

(1) This is analogous to the connection representation in which the "triad density" is represented by the functional derivative $-\underline{\vartheta}^{\alpha}:=\delta / \delta \underline{A}_{\alpha}^{ \pm}$with respect to the canonically conjugate Ashtekar variable $\underline{A}_{\alpha}^{ \pm}$. This provides a mapping from the Hamiltonian constraint of gravity with cosmological term to the Chern-Simons 3-form [7] of the Ashtekar-Sen connection. 
a Möbius type representation $[9,10]$. The $A(n, R)$ is that subgroup of $G L(n+1, R)$ which leaves the $n$-dimensional hyperplane $\overline{\bar{R}}^{n}:=\left\{\overline{\bar{x}}=\left(\begin{array}{l}x \\ 1\end{array}\right) \in R^{n+1}\right\}$ invariant:

$$
A(n, R)=\left\{\left(\begin{array}{ll}
\Lambda & \tau \\
0 & 1
\end{array}\right) \in G L(n+1, R) \mid \Lambda \in G L(n, R), \tau \in R^{n}\right\} .
$$

Thus we obtain $\overline{\bar{x}}^{\prime}=\left(\begin{array}{c}\Lambda x+\tau \\ 1\end{array}\right)$ as it is required for the action of the affine group on the flat affine space.

The Lie algebra $a(n, R)$ consists of the generators $P_{\gamma}$ representing local $\mathrm{n}$-dimensional translations and the $L^{\alpha}{ }_{\beta}$ which span the Lie algebra $g l(n, R)$ of $n$-dimensional linear transformations. Their commutation relations are:

$$
\begin{gathered}
{\left[P_{a}, P_{3}\right]=0,} \\
{\left[L_{\beta}^{a}, P_{\gamma}\right]=\delta_{\gamma}^{\alpha} P_{\beta},}
\end{gathered}
$$

and

$$
\left[L_{\beta}^{a}, L_{\delta}^{\gamma}\right]=\delta_{\delta}^{a} L_{\beta}^{\gamma}-\delta_{\beta}^{\gamma} L_{\delta}^{a} .
$$

Observe that the physical dimensions of these generators are $\left[L^{\alpha}{ }_{\beta}\right]=\hbar$ and $\left[P_{\alpha}\right]=$ $\hbar /$ length. If there is a spacetime metric $g=g_{\alpha \beta} \vartheta^{\alpha} \otimes \vartheta^{\beta}$ with components $g_{\alpha \beta}$ available for lowering indices, the general linear group can be decomposed further:

$$
L_{\alpha \beta}=\left(\stackrel{\circ}{L}_{\alpha \beta}+\not{ }_{\alpha \beta}+\frac{1}{n} g_{\alpha \beta} \mathcal{D}\right) \text {. }
$$

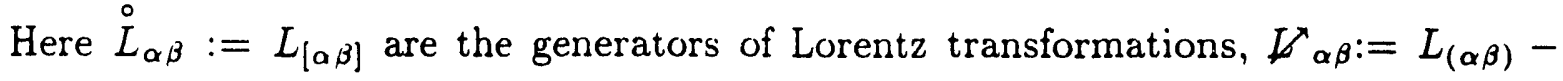
$(1 / n) g_{\alpha \beta} L^{\gamma}$, represent shears, whereas $\mathcal{D}:=L_{\gamma}^{\gamma}$ is the generatcr of scale transformations.

\section{The affine gauge approach}

In a matrix representation analogous to (2.1), we can write the affine gauge $\operatorname{group}^{(2)}$ as

$$
\mathcal{A}(n, R)=\left\{\left(\begin{array}{cc}
\Lambda(x) & \tau(x) \\
0 & 1
\end{array}\right) \mid \Lambda(x) \in \mathcal{G} \mathcal{L}(n, R), \quad \tau(x) \in \mathcal{T}(n, R)\right\}
$$

(2) In a fibre bundle approach, one introduces first the bundle of affine frames $A(M):=P\left(M^{n}, A(n, R), \pi, \delta\right)$ where $\pi$ denotes the projection to the base manifold and $\delta$ the (left) action of the structure group $A(n, R)$ on the bundle. Active, affine gauge transformations are the vertical automorphisms of $A(M)$. Similarly as the diffeomorphisms of the base manifold $M^{n}$, they form the infinite-dimensional 
Following a Yang-Mills type gauge approach, we introduce the generalized affine connection [14], cf. [15],

$$
\approx=\left(\begin{array}{cc}
\Gamma^{(L)} & \Gamma^{(T)} \\
0 & 0
\end{array}\right)=\left(\begin{array}{cc}
\Gamma_{\alpha}^{(L) \beta} L_{\beta}^{\alpha} & \Gamma^{(T) \alpha} P_{\alpha} \\
0 & 0
\end{array}\right)
$$

and require that it transforms inhomogeneously under an affine gauge transformation:

$$
\approx \stackrel{A^{-1}(x)}{\longrightarrow} \underset{\tilde{\Gamma}}{\tilde{\Gamma}^{\prime}}=A^{-1}(x) \widetilde{\widetilde{\Gamma}} A(x)-A^{-1}(x) d . A(x), \quad A(x) \in \mathcal{A}(n, R) .
$$

Since we regard it as an active transformation, it is formed with respect to the group element

$$
A^{-1}(x)=\left(\begin{array}{cc}
\Lambda^{-1}(x) & -\Lambda^{-1}(x) \tau(x) \\
0 & 1
\end{array}\right)
$$

which is inverse to $A(x) \in \mathcal{A}(n, R)$. The corresponding affine curvature is given by

$$
\approx \widetilde{\widetilde{R}}:=d \tilde{\Gamma}+\widetilde{\widetilde{\Gamma}} \wedge \approx \widetilde{\Gamma}=\left(\begin{array}{cc}
d \Gamma^{(L)}+\Gamma^{(L)} \wedge \Gamma^{(L)} & d \Gamma^{(T)}+\Gamma^{(L)} \wedge \Gamma^{(T)} \\
0 & 0
\end{array}\right)
$$

and transforms covariantly, i.e.

$$
\widetilde{\widetilde{R}} \stackrel{A^{-1}(x)}{\longrightarrow} \underset{\widetilde{\widetilde{R}}^{\prime}}{\longrightarrow}=A^{-1}(x) \widetilde{\widetilde{R}} \cdot A(x)
$$

under the affine gauge group. ${ }^{(3)}$ The covariant exterior derivative $\widetilde{\widetilde{D}}:=d+\widetilde{\widetilde{\Gamma}}$ acts on an affine p-form $\tilde{\widetilde{\Psi}}=\left(\begin{array}{c}\Psi \\ 1\end{array}\right)$ as follows

$$
\approx \tilde{D} \tilde{\Psi}=\left(\begin{array}{c}
D \Psi+\Gamma^{(T)} \\
0
\end{array}\right)
$$

group $\mathcal{A}(n, R):=C^{\infty}\left(A(M) \times_{A d} A(n, R)\right)$. The group $\mathcal{G} \mathcal{L}(n, R):=C^{\infty}\left(A(M) \times_{A d}\right.$ $G L(n, R)$ ) of linear gauge transformations and the group $\mathcal{T}(n, R):=C^{\infty}\left(A(M) \times_{A d}\right.$ $R^{n}$ ) of local translations are subgroups of $\mathcal{A}(n, R)$. Taking the cross-section in the associated bundle is abbreviated by $C^{\infty}$ and $A d$ denotes the adjoint representation with respect to $G L(n, R)$. Due to its construction, the group of local translations $\mathcal{T}(n, R)$ is locally isomorphic to the group of active diffeomorphisms $\operatorname{Diff}(n, R)$ of the manifold [11. 12]. The infinite-dimensional group $\operatorname{Dif} f(n, R)$ contains the $n+n^{2}$ -dimensional group $A(n, R)_{H}$ of holonomic affine transformations [13] which are generated by the vector fields $P_{i}=\partial_{i}:=\partial / \partial x^{i}$ and $L^{i}{ }_{j}=x^{i} \partial_{j}$ as a subgroup. Note that differentiable coordinate transformations, which leave exterior forms invariant, are regarded as passive diffeomorphisms.

(3) Our matrix formalism, cf. $[10,16]$ and references therein, is a spacetime generalization of the so-called motor calculus of von Mises [17]. 
Only by imposing the gauge $\Gamma^{(T)}=0$, one would recover the covariant exterior derivative $D:=d+\Gamma^{(L)}$ with respect to the linear connection.

After insertion of (3.2) and (3.4), the inhomogeneous transformation law (3.3) splits into

$$
\Gamma^{(L)} \stackrel{A^{-1}(x)}{\longrightarrow} \Gamma^{(L) \prime}=\Lambda^{-1}(x) \Gamma^{(L)} \Lambda(x)-\Lambda^{-1}(x) d \Lambda(x),
$$

and

$$
\Gamma^{(T)} \stackrel{A^{-1}(x)}{\longrightarrow} \Gamma^{(T) \prime}=\Lambda^{-1}(x) \Gamma^{(T)}-\Lambda^{-1}(x) D \tau(x) .
$$

The local translations $\tau(x)$ automatically drop out in (3.8) due to the 1-form structure of $\Gamma^{(T)}$. Thereby (3.8) aquires the conventional transformation rule (with the exterior derivative $d$ ) for a Yang-Mills-type connection for $\mathcal{G L}(n, R)$, and thus we can identify $\Gamma^{(L)}=\Gamma=\Gamma_{\alpha}{ }^{\beta} L^{a}{ }_{\beta}$ with the linear connection. Due to the covariant exterior derivative term $D \tau(x):=d \tau\left(x^{\prime}\right)-\Gamma^{(L)} \tau(x)$ in (3.9), the translational part $\Gamma^{(T)}$ does not transform as a covector as is required for the coframe $\vartheta:=\vartheta^{\alpha} P_{\alpha}$, i.e. the 1-form with values in the Lie algebra of $R^{n}$.

However, we may follow Trautman [18] and introduce a vector-valued 0 -form $\tilde{\widetilde{\xi}}=\left(\begin{array}{l}\xi \\ 1\end{array}\right)=\left(\begin{array}{c}\xi^{\alpha} P_{\alpha} \\ 1\end{array}\right)$ which transforms as $\widetilde{\tilde{\xi}}^{\prime}=A^{-1}(x) \approx \tilde{\xi}$, i.e.,

$$
\xi \stackrel{A^{-1}(x)}{\longrightarrow} \xi^{\prime}=\Lambda^{-1}(x)(\xi-\tau(x))
$$

under an active affine gauge transformation. Then

$$
\vartheta:=\Gamma^{(T)}-D \xi
$$

transforms as a vector-valued 1 -form under the $\mathcal{A}(n, R)$, as required:

$$
\vartheta \stackrel{A^{-1}(x)}{\longrightarrow} \vartheta^{\prime}=\Lambda^{-1}(x) \vartheta
$$

If $\Gamma^{(T)}$ vanished throughout the manifold, the vector field $\xi$ would represent a four dimensional version of Cartan's generalized radius vector [19]. The integrability condition is, in this instance, given by the vanishing of the translational part of the affine curvature (3.5), i.e., $R^{(T)}:=D \Gamma^{(T)}=\left(T^{\alpha}+R_{\beta}^{(L) \alpha} \xi^{\beta}\right) P_{\alpha}=0$, whirh, for teleparallelism models with $R_{a}^{(L) \beta}=0$, would imply vanishing torsion. ${ }^{(4)}$

(4) Otherwise there also exists, for example, the nontrivial solution $T^{\alpha}=c \eta^{\alpha \beta} \xi_{\beta}$ and $R^{\alpha \beta}=\mathrm{C \eta} \eta^{\alpha \beta}$. rith the dimensionful constant c. 


\section{Reduction to a Cartan comnection}

Our key relation (3.11) in components takes the form

$$
\Gamma_{i}^{(T) a}=\epsilon_{i}{ }^{\alpha}+D_{i} \xi^{\alpha},
$$

which for $D_{i} \xi^{\alpha}=-\delta_{i}^{\alpha}$ makes contact with an approach of Hayashi et al. [20]. In a recent paper [21] on the Poincaré gauge approach, our $\xi^{\alpha}$ are kinematically interpreted as "Poincaré coordinates"; note that in Eq. (2.14) of that paper [21], vielbein and translational connection are identified opposite to our notation. Observe also that we do not have to put the "Poincaré coordinates" $\xi^{\alpha}$ to zero, in order to obtain the gauge aftine (!) transformation law (3.12) of the coframe. The reason is that the local translations are now "hidden" in the invariant transformation behavior of the exterior 1-form $\vartheta$ under (passive) diffeomorphisms. Note also that in our approach, in contradistinction to that of Sexl and Urbantke [22, p. 381], we do not need to break the affine gauge group kinematically via $D_{\tau}(x)=0$. An attempt to motivate the translational connection (4.1) from the theory of dislocations can be found in Ref. [23]. whereas Hennig and Nitsch [24] provide an explanation in terms of jet bundles.

Since $\xi=\xi^{a} P_{\alpha}$ aquires its values in the "orbit" (coset space) $A(n, R) / G L(n, R) \approx$ $R^{n}$, it can be regarded as an affine vector field (or "generalized Higgs field" according to Trautman [25]) which "hides" the action of the local translational "symmetry" $\mathcal{T}(n, R)$. If we required [26] the condition

$$
D \xi=0,
$$

the translational connection $\Gamma^{(T)}$ would, together with the coframe $\vartheta$, be "soldered" to the spacetime manifold, cf. [27], and the translational part of the local affine group would be "spontaneously broken", cf. [28]. The stronger constraint of a "zero section" vector field $\xi=0$ would reduce the generalized affine connection $\tilde{\tilde{\Gamma}}$ on the affine bundle. $\mathcal{A}(M)$ to the Cartan connection [9]

$$
\overline{\bar{\Gamma}}=\left(\begin{array}{ll}
\Gamma & \vartheta \\
0 & 0
\end{array}\right)
$$

on the bundle $L(M)$ of linear frames. Due to (3.12), this is not anymore a connection in the usual sense. However, thereby we would recover the familiar (metric-)affine geometrical arena [29] with nonmetricity, torsion, and curvature, as is summarized in the following table:

\begin{tabular}{|c|c|c|}
\hline potential & field strength & Bianchi identity \\
\hline metric $g^{\alpha \beta}$ & $Q^{\alpha \beta}=D g^{\alpha \beta}$ & $D Q^{\alpha \beta}=2 R_{\mu}{ }^{\alpha} g^{\beta) \mu}$ \\
coframe $\vartheta^{\alpha}$ & $T^{\alpha}=D \vartheta^{\alpha}$ & $D T^{\alpha}=R_{\mu}{ }^{\alpha} \wedge \vartheta^{\mu}$ \\
connection $\Gamma_{\alpha}{ }^{\beta}$ & $R_{\alpha}{ }^{\beta}=d \Gamma_{\alpha}{ }^{\beta}+\Gamma_{\mu}{ }^{\beta} \wedge \Gamma_{\alpha}{ }^{\mu}$ & $D R_{\alpha}{ }^{\beta}=0$ \\
\hline
\end{tabular}




\section{j. Affine gauge transformations versus active diffeomorphisms}

The affine gauge transformations in (3.3) are finite transformations. If we expand them up to first order according to

$$
\begin{gathered}
\Lambda(x)=1+\omega_{a}{ }^{\beta} L^{\alpha}{ }_{\beta}+\cdots, \\
\tau(x)=0+\varepsilon^{a} P_{\alpha}+\cdots,
\end{gathered}
$$

we obtain from (3.8) and (3.9), respectively,

$$
\begin{gathered}
\delta_{A^{-1}} \Gamma^{(L)}=-\left(D \omega_{\alpha}{ }^{\beta}\right) L^{\alpha}{ }_{\beta}+\cdots, \\
\delta_{A^{-1}} \Gamma^{(T)}=-\left(D \varepsilon_{\alpha}+\omega_{\beta}{ }^{a} \Gamma^{(T) \beta}\right) P_{\alpha}+\cdots .
\end{gathered}
$$

(For the "product" of Lie generators we use the Lie brackets of Sect. 2, since we work in the adjoint representation.) It is gratifying to note that the leading exterior covariant derivatives reveal, in particular, that the translational connection $\Gamma^{(T)}$ is really the "compensating" field for infinitesimal local translations $\varepsilon$ in the Yang-Mills sense.

Let us compare this result with the "diffeomorphism" approach, which was orginally developed for the Poincaré subgroup of the $A(n, R)$ : In essence, the translational part of the transformation

$$
\Pi=1-\varepsilon-\omega=1-\varepsilon^{\alpha} P_{\alpha}-\omega_{\alpha}^{\beta} L^{\alpha}{ }_{\beta}
$$

is embedded as an $n$-parameter subgroup of the infinite-dimensional group of active diffeomorphisms of spacetime. ${ }^{(5)}$ In order to calculate the effect on the linear conection and the coframe, one has to consider the action [30] of the Lie derivative $\mathcal{L}_{(-\varepsilon)}$ with respect to the vector field $(-\varepsilon)$ together with an infinitesimal frame rotation paiametrized by $(-\omega)$. Since $\left.\left.\mathcal{L}_{(-\varepsilon)}=\ell_{(-\varepsilon)}:=-(\varepsilon\rfloor d+d \varepsilon\right\rfloor\right)$ holds for geometrical objects which are invariant under changes of the basis, a straightforward calculation yields

$$
\left.\left.\left(\mathcal{L}_{(-\varepsilon)}+\delta_{(-\omega)}\right) \Gamma=-\left[D\left(\omega_{\alpha}{ }^{\beta}+\varepsilon\right] \Gamma_{\alpha}{ }^{\beta}\right)+\varepsilon\right\rfloor R_{\alpha}{ }^{\beta}\right] L^{\alpha}{ }_{\beta}
$$

and

$$
\left.\left.\left(\mathcal{L}_{(-\varepsilon)}+\delta_{(-\omega)}\right) \vartheta=-\left[D \varepsilon^{\alpha}-\left(\omega_{\beta}{ }^{\alpha}+\varepsilon\right] \Gamma_{\beta}{ }^{\alpha}\right) \vartheta^{\beta}+\varepsilon\right] T^{\alpha}\right] P_{\alpha}
$$

The 'annoying' linear connection term in (5.6) and (5.7) can be dismissed by going over to the parallel transport version of Hehl et al. [2] and Ne'eman [31] in

(5) The minus sign is in accordance with our earlier conventions for active transformations. 
which, instead of $P_{\alpha}$, the covariant derivative components $\left.D_{\alpha}:=e_{\alpha}\right\rfloor D$ are adopted as generaturs of local translations: Then the infinitesimal transformations read

$$
\left.\check{\Pi}=1-\varepsilon^{\alpha} D_{\alpha}-\omega_{\alpha}{ }^{\beta} L^{\alpha}{ }_{\beta}=\Pi+\varepsilon\right\rfloor \Gamma_{\alpha}{ }^{\beta} L^{\alpha}{ }_{\beta} .
$$

Since this amounts to a redefinition $\tilde{\omega}:=\omega-\varepsilon \mid \Gamma_{\alpha}{ }^{\beta} L^{\alpha}{ }_{\beta}$ of the parameters of the infinitesimal linear transformation, we can simply read off, from (5.6) and (5.7), the new results

$$
\left.\left(\mathcal{L}_{(-\varepsilon)}+\delta_{(-\dot{\omega})}\right) \Gamma=-\left[D \omega_{\alpha}{ }^{\beta}+\varepsilon\right] R_{\alpha}{ }^{\beta}\right] L^{\alpha}{ }_{\beta},
$$

and

$$
\left.\left(\mathcal{L}_{(-\varepsilon)}+\delta_{(-\dot{\omega})}\right) \vartheta=-\left[D \varepsilon^{\alpha}-\omega_{\beta}{ }^{\alpha} \vartheta^{\beta}+\varepsilon\right] T^{\alpha}\right] P_{\alpha} .
$$

In this parallel transport version, the leading covariant derivative pieces are the same as in the affine gauge approach. In particular, the "hidden" translational piece in the affine transformation (3.12) of the coframe gets thereby "uncovered" in (5.10). Is it in the end "...somewhat a matter of taste...", as Nester [32] has put it, whether or not one prefers the parallel transport interpretation of translations over the affine gauge approach? One could argue that the Pauli-type curvature and torsion terms in the infinitesimal transformations (5.9) and (5.10) violate the spirit of the principle of "minimal coupling", a cornerstone of a conventional Yang-Mills type gauge approach. These terms also show up in the commutation relation

$$
\left[D_{\alpha}, D_{\beta}\right]=-T_{\alpha \beta}{ }^{\top} D_{\gamma}+R_{\alpha \beta \mu}{ }^{\nu} L^{\mu}{ }_{\nu}
$$

for the operator $D_{\alpha}$ of parallel-transport, if applied to a 0 -form. Due to the torsion and curvature terms on the right-hand side, a softening [33] of the Lie algebra structure cannot be avoided in such a diffeomorphism-type approach. Using the covariant derivatives (or Lie derivatives) has the advantage of being physically meaningful as a parallel transport, as explained in Ref. [2], once we put up a frame, and, in a corresponding first order approach, these 'non-minimal' structures do not touch the explicit form of the Lagrangian. However, they' are algebraically less useful because (5.11) is not a Lie algebra any more. Moreover, as we will show below, the affine gauge approach lends itself to an important resolution of degeneracy problems in quantum gravity. 


\section{Affine gauge approach to quantum gravity with topology change}

Now we may return to the question of the proper meaning and range of validity of the functional integral (1.2) in quantum gravity. The lesson learnt from our affine gauge approach is that, instead of the coframe, a summation over the true translational connection $\Gamma^{(T)}$ is more akin to a quantum Yang-Mills type approach. Moreover. it would cause no problems if the functional integration didn't go only through $\Gamma^{(L)}=0$ as it would do in Yang-Mills theory, but through $\Gamma^{(T)}=0$ as well, keeping the coframe $\vartheta$, by definition, non-degenerate. In effect, we may now consider the functional integral

$$
\int \mathcal{D} \tilde{\tilde{\Gamma}} \exp \left(i \int_{M} V_{\mathrm{EC}}\right)
$$

where summation is understood over the generalized affine connection.

Due to (3.8) and (3.12), the Einstein-Cartan Lagrangian is gauge invariant also with respect to the full, but "hidden", affine gauge group $\mathcal{A}(4, R)$. In order to study the possibility of a degenerate or even vanishing translational connection, it is instructive to insert the representation (3.11) of the coframe into the Einstein-Cartan Lagrangian (1.1) amended by a cosmological term $\left(-\Lambda_{\cos } / \ell^{2}\right) \eta$. In the gauge $\Gamma^{(T)} \rightarrow 0$, we finci then the following truncated expression:

$$
\begin{aligned}
& V_{\mathrm{ECC}}=-\frac{1}{4 \ell^{2}} \stackrel{\circ}{R^{\alpha \beta}} \wedge\left(\Gamma^{(T) \gamma}-D \xi^{\gamma}\right) \wedge\left(\Gamma^{(T) \delta}-D \xi^{\delta}\right) \eta_{\alpha \beta \gamma \delta} \\
& -\frac{._{\cos }}{4 ! C^{2}}\left(\Gamma^{(T) \alpha}-D \xi^{\alpha}\right) \wedge\left(\Gamma^{(T) \beta}-D \xi^{\beta}\right) \wedge\left(\Gamma^{(T) \gamma}-D \xi^{\gamma}\right) \wedge\left(\Gamma^{(T) \delta}-D \xi^{\delta}\right) \eta_{\alpha \beta \gamma \delta} \\
& \longrightarrow \quad \frac{1}{4 \ell^{2}} \stackrel{\circ}{R}^{\alpha \beta} \wedge \xi^{\gamma} \stackrel{\circ}{R}_{\mu}^{\delta} \xi^{\mu} \eta_{\alpha \beta \gamma \delta}+\frac{\Lambda_{\cos }}{4 ! \ell^{2}} \xi^{\alpha} D \xi^{\beta} \wedge D \xi^{\gamma} \wedge \stackrel{\circ}{R}_{\mu}{ }^{\delta} \xi^{\mu} \eta_{\alpha \beta \gamma \delta} \\
& -\frac{1}{4 \ell^{2}} d\left[\left(\stackrel{\circ}{R}^{\alpha \beta} \wedge \xi^{\gamma} D \xi^{\delta}+\frac{\Lambda_{\cos }}{3 ! \ell^{2}} \xi^{\alpha} D \xi^{\beta} \wedge D \xi^{\gamma} \wedge D \xi^{\delta}\right) \eta_{\alpha \beta \gamma \delta}\right] .
\end{aligned}
$$

In order to separate off the boundary term, we employed the Bianchi identity $D \stackrel{\circ}{R}^{\alpha \beta}=$ 0 for the Riemann-Cartan curvature, which is valid in the Riemann-Cartan framework of (6.2) with vanishing nonmetricity.

Thus the occurrence of a vanishing translational connection in the functional integral (6.1) is without harm, since a quadratic and linear curvature Lagrangian remains for the linear connection. In case that $\Lambda_{\cos }=0$, except for the dimensional coupling constant, the truncated expression (6.2) resembles the Stephenson-Kilmister-Yang ( $\mathrm{SKY}^{Y}$ ) Lagrangian $[34,35,36]$, which is known to be perturbatively renormalizable 
[37. 38]. Moreover, for the constant vacuum condensate $\xi_{<>}^{0}=H \neq 0$ and zero otherwise, the Lagrangian (6.2) reduces "spontaneously" to the Euclidean 3D topological gravity model

$$
V_{\mathrm{ECC}<>}=\frac{1}{4 \ell^{2}}\left[\eta_{A B C} \stackrel{\circ}{R}^{A B} \wedge{\stackrel{\circ}{R_{\dot{0}}}}^{C} H^{2}+\frac{\Lambda_{\mathrm{cos}}}{3 !} \eta_{A B C} \stackrel{\circ}{\Gamma}_{\dot{0}}^{A} \wedge \stackrel{\circ}{\Gamma}_{\dot{0}}^{B} \wedge \stackrel{\circ}{\Gamma}_{\dot{0}}^{C} H^{4}\right]
$$

in which the dreibein can be identified with part of the Lorentz connection via $\vartheta^{A}=$

$\stackrel{\circ}{\Gamma}_{\dot{0}}{ }^{A}$ and $T^{A}=\stackrel{\circ}{R}_{\dot{0}}{ }^{A}$. Other directions of symmetry breaking, such as $\xi_{\langle>}^{\dot{3}}=H \neq 0$, lead to Minkowskian models of $3 \mathrm{D}$ gravity [39], cf. [40]. For a time-dependent $H=H(t)$, one would obtain, in addition to the curvature dependent $H^{2}+H^{4}$ potential in (6.3), a kinetic term for $H$ and could analyse, following Giddings [41], the instability of the $\xi^{\alpha}=0$ solution. In the gauge $\Gamma^{L}=0$, we thereby obtain a means to analyse the instability not only for the diffeomorphism invariant solution $\vartheta=0$ but also for the true translation invariant solution $\Gamma^{T}=0$. It remains to be seen, if also the signature of the physical spacetime has a dynamical origin in such a framework, as is suggested by Greensite [42]: cf. [43].

In quantum gravity, a vanishing translational connection $\Gamma^{(T)}$ may be accompanied by a topology change of the underlying spacetime manifold. The rich spectrum of possible topological structures in quantum "geometrodynamics" has been outlined in Ref. [44]. Our affine gauge approach exactly leads us to the more detailed mechanism devised by Horowitz [3]. According to (4.11), the vanishing of the translational connection converts the coframe components into the form $\vartheta^{\alpha}=-D \xi^{\alpha}$. At each of the boundaries $\partial M^{4} \approx S^{3} \cup S^{\prime 3}$ of the topology changing spacetime manifold $M^{4}$ we may also adopt the gauge $\Gamma^{(L)}=0$ for the linear (or Lorentz) connection. In the vicinity of that boundary,

$$
\vartheta^{\alpha}=-d \xi^{\alpha} \quad \text { and } \quad g=o_{\alpha \beta} d \xi^{\alpha} \otimes d \xi^{\beta}
$$

represent the (flat !) Minkowskian spacetime in terms of the 4-dimensional Cartesian coordinate system $\left\{\xi^{\alpha}\right\}$. Obviously, this solves also the vacuum Einstein-Cartan theory with zero action. For generic $\xi^{\alpha}$, the (inverse) tetrad components $e_{j}^{\beta}=-\partial_{j} \xi^{\beta}$ will be non-degenerate almost everywhere. In order to accomplish a spatial topology change, we may choose $\xi^{0}$ to be the "height function" of Morse theory. Then, $d \xi^{0}$ is a timelike covector and we can apply the "trouser world" construction as is decribed, for example, by Konstantinov and Melnikov in Fig. 1 of Ref. [45].

We summarize: The advantage of the affine gauge approach is that the coframe, even for vanishing translational connection, remains non-degenerate almost everywhere.

\section{Acknowledgments}

We would like to thank Jörg Hennig (Clausthal) and Yuri Obukhov (Cologne/ Moscow) for constructive comments on a preliminary version of this paper. 


\section{References}

[1] R.P. Feynman: Lectures on Gravitation. Lecture notes by F.B. Morinigo and W.G. Wagner (California Institute of Technology, Pasadena, California 1962/63).

[2] F. Hehl, P. v. Heyde, G.D. Kerlick and J.M. Nester, Rev. Mod. Phys. 48, 393 (1976); see also F.W. Hehl: "Four lectures on Poincaré gauge theory", in: Proceedings of the 6th Course of the School of Cosmology and Gravitation on Spin. Torsion, Rotation, and Supergravity, held at Erice, Italy, May 1.979, P. G. Bergmann, V. de Sabba'a, eds. (Plenum, New York 1980), p.5.

[3] G.T. Horowitz, Class. Quantum Grav. 8 (1991) 587.

[4] C.J. Isham: "Conceptual and geometrical problems in quantum gravity", in: Re. cent Aspects of Quantum Fields, Proceedings of the $\mathrm{XXX}^{\text {th }}$ Int. Universitätswochen für Kernphysik, Schladming, Austria, February and March 1991, H. Mitter and H. Gausterer, eds., Lecture Notes in Physics, Vol. 396 (Springer, Berlin $1991)$, p.123.

[5] E.W. Mielke, Ann. Phy's. (N.Y.) 219 (1992) 78.

[6] P. Baekler, E.W. Mielke, and F.W. Hehl, Nuovo Cimento 107B (1992) 91.

[i] B. Brügmann. R. Gambini. and J. Pullin, Nucl. Phys. B 385 (1992) 587.

[8] E. Witten, Nucl. Phys. B311 (1988/89) 46.

[9] S. Kobayashi: Transformation Groups in Differential Geometry (Springer, New York 1972).

[10] E.W. Mielke: Geometrodynamics of Gauge Fields - On the geometry of YangMills and gravitational gauge theories (Akademie-Verlag, Berlin 1987).

[11] V.I. Ogievetsky, Lett. Nuovo Cimento 8 (1973) 988.

[12] S. Sternberg, Ann. Phys. (N.Y.) 162 (1985) 85.

(13] P.G. Bergmann and A.B. Komar, J. Math. Phys. 26 (1985) 2030.

[11] S. Kobayashi and K. Nomizu: Foundations of Differential Geometry, Vol. I (Interscience Publ., New York 1963). 
[15] L.K. Noris, R.O. Fulp, and W.R. Davies. Phys. Lett. 79A (1980) 278.

[16] K.L. Malyshev and A.E. Romanov: "Aspects of $T(3) \& S O(3)$-gauge theory of dislocations and disclinations I and II", in Russian, Leningrad preprints LOMI P-2-90 and P-5-90 (1390).

[17] R. von Mises: "Motorrechnung, ein neues Hilfsmittel der Mechanik", ZAMM 4 (1924) 155.

[18] A. Trautman: "On the structure of the Einstein-Cartan equations", in: Differen ial Geometry, Symposia Matematica Vol.12 (Academic Press, London 1973) p.139.

[19] E. Cartan: On a manifold with an affine connection and the theory of general relativity (Bibliopolis, Napoli 1986).

[20] I. Hayashi and T. Nakano. Progr. Theor. Phy's. 38 (1967) 491; K. Hayashi and T. Shirafuji. Progr. Theor. Phys. 64 (1980) 866; 80 (1988) 711.

[21] G. Grignani and G. Nardelli, Phys. Rer. 45 (1992) 2719.

[22] R.U. Sexl and H.K. Urbantke: Gravitation und Kosmologie, 2nd edition (Bibliographisches Institut, Mannheim 1983).

[23] G. Sardanashvily and M. Gogbershvily, Mod. Phys. Lett. A2 (1987) 609.

[24] J. Hennig and J. Nitsch, Gen. Rel. Grav. 13 (1981) 947.

[25] A. Trautman, Czech. J. Phy's. B29 (1979) 107.

[26] I.A. Pilch, Lett. Math. Phys. 4 (1980) 49.

[27] P.K. Smrz, J. Math. Phys. 28 (198i) 2824.

[28] L. O'Raiffeartaigh: "Jome hidden aspects of hidden symmetry", in: Differential Geometry, Group Representations, and Quantization, Lecture Notes in Physics, Vol. 379. J.D. Hennig, W. Lücke, and J. Tolar, eds. (Springer, Berlin 1991), p. 99.

[29] F.W. Hehl. J.D. McCrea, E.W. Mielke, and Y. Ne'eman, Found. Phys. 19, 1075 (1989); Phys. Rep. (to be published); J.D. McCrea, Class. Quantum Grav. 9, $553(1992)$. 
[30] R.D. Hecht. F.W: Hehl, J.D. McC'rea. E.W: Mielke, and Y. Ne'eman: "Improved enelgy-momentum currents in metric-affine spacetime". Phys. Lett. A (in print 1992).

[31] Y. Ne'eman, in: Differential Geometrical Methods in Mathematical Physics, I. Bleuler, H. R. Petry, and A. Reetz. eds. Lecture Notes in Mathematics, Vol. 676 (Springer-Verlag, Berlin 1978), p.189.

[32] J. M. Nester, in: An Introduction to Kaluza-Klein Theories, Workshop Chalk River/Deep River, Ontario 11-16 Aug. 1983, H. C. Lee, eds. (World Scientific, Singapore 1984), p.83.

[33] T.W.B. Kibble and K.S. Stelle: "Gauge theories of gravity and Supergravity", in: Progress in Quantum Field Theory: Festschrift for Umezawa. H. Ezawa and S. Iiamefuchi. eds. (Elsevier Science Publ. B.V. 1986), p. 57.

[34] G. Stephenson: Nuovo Cimento 9 (1958) 263.

[35] C'.W. Kilmister and D..J. Newman, Proc. Cambridge Phil. Soc. (Math. Phys. Sc.) 57 (1961) 851.

[36] C..N. Yang, Phys. Rev. Let.t. 33 (1974) 445.

[3i] K.S. Stelle, Phys. Rev. D16 (1977) 953.

[38] C.-Y. Lee and Y. Ne'eman, Phys. Lett. B242 (1990) 59; C.-Y. Lee, Class. Quantum Grav. 9 (1992) 2001.

[39] T.T. Burwick, A.H. Chamseddine, and K.A. Meissner, Phys. Lett. B284 (1992) 11.

[40] G. Grignani and G. Nardelli: "Chern-Simons gravity from $3+1$ dimensional gravity", preprint DFUPG-57-9?.

[41] S.B. Giddings, Phys. Lett. B268 (1991) 17.

[42] J. Greensite. "Dynamical origin of the Lorentzian signature of spacetime", preprint NBI-HE-92-59.

[43] A.D. Sakharov, Sov. Phys. JETP 60 (1985) 214.

[44] E.W. Mielke: Gen. Rel. Grav. 8 (1977) 175.

[4i] M.Yu. Fonstantinov and V.N. Melnikov: Class. Quantum Grav. 3 (1986) 401. 

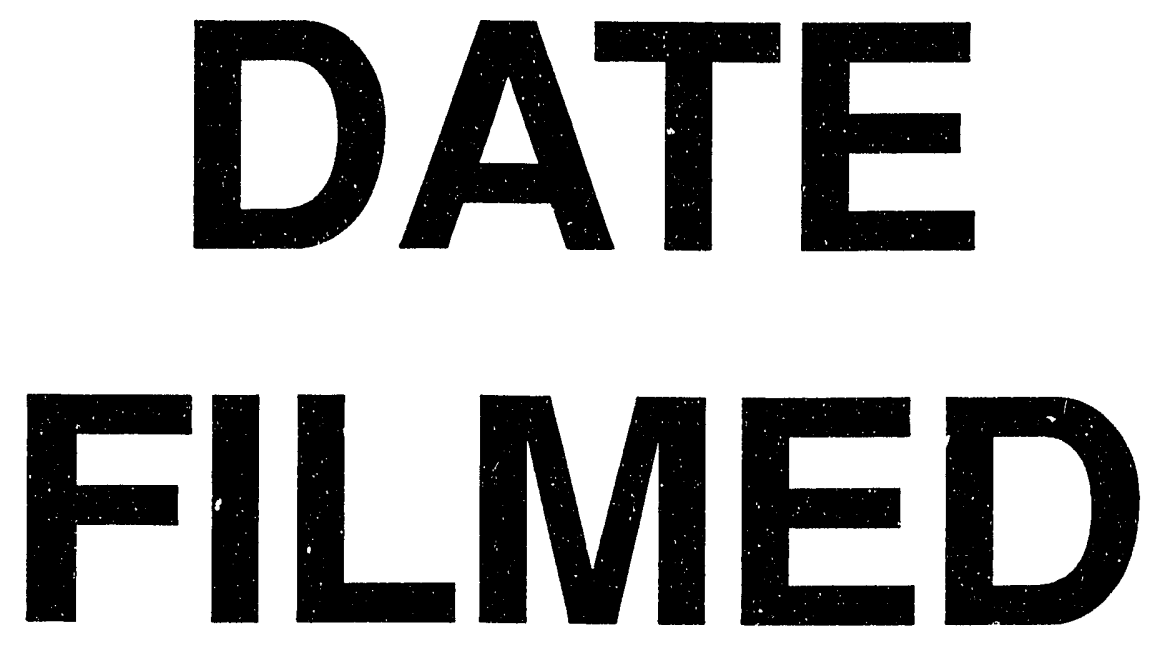

$6 / 17 / 94$
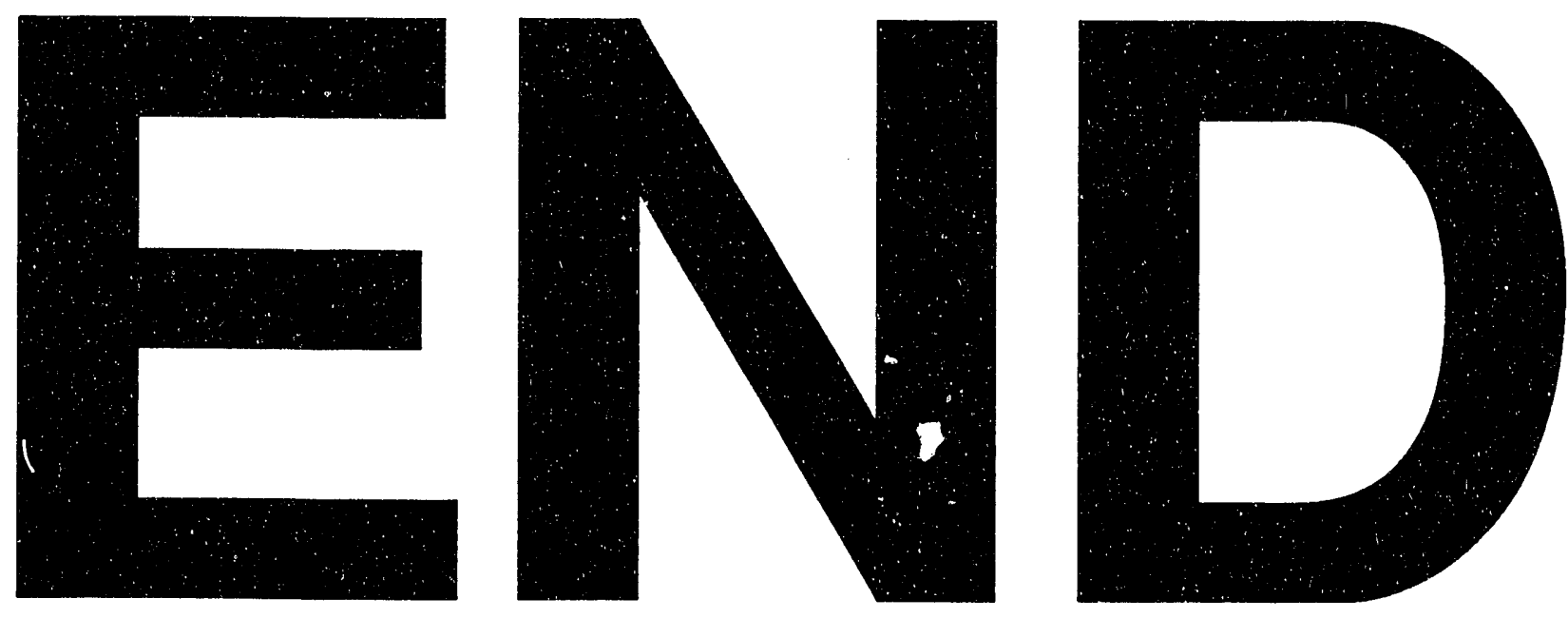
